\title{
Things, tags, topics: Thingiverse's object-centred network
}

Robbie Fordyce, Luke Heemsbergen, Thomas Apperley, Michael

Arnold, Thomas Birtchnell, Michael Luo \& Bjorn Nansen

\section{Introduction}

This paper reports on the application of social network analysis (SNA) methods to aggregate metadata from the 3D printing file-sharing website, Thingiverse. Thingiverse claims to be the largest repository of 3D printable user-created objects and offers the exemplar case to explore the emerging cultures of consumer 3D printing. By applying networking principles to records of connections via 'tags' shared between items uploaded by users to Thingiverse, it is possible to map out the use of tags in a way that identifies habits of use of the site and methods of understanding by users of the site. From this, we make some specific observations about the practice of 3D printing via how objects bind the community of Thingiverse, and begin to identify analytical frameworks for further research where sociality is mediated by specific digital objects and their metadata. Of importance to the study of methodology vis-avis digital research, we are interested in reflecting on how the opportunities and constraints of metadata offer both specific modes of social organisation and specific modes of enquiry for researchers. Tagging is often linked to practices of folksonomy, but such organisation may be more accurately understood by a concept we tentatively introduce below as ontosonomy ${ }^{1}$ : where people, things and networks work towards distribution, arrangement, and management of knowledge. We speculate on these theoretical concerns as way of conclusion.

\footnotetext{
${ }^{1}$ The Oxford English Dictionary does not list Ontosomy, and we apologize for submitting to use of the term here. An introduced neologism is rarely worth its merit, but in this case we hope our research speaks to the material reality of how people, objects and networks create and distribute order in digital spaces in a way that begs a recognizable term.
} 
Our empirical data, and current interest in Thingiverse, builds from research done by Jarkko Moilanen, Angela Daly, Ramon Lobato, and Darcy Allen (2015), which both analyses Thingiverse, and critiques its intellectual property policies. In order to address these concerns, Moilanen et al. report a website-scraping project which attempts to map out the licencing choices by users of Thingiverse. The observations from their research note the profound contradictions and intellectual property tensions and disputes within Thingiverse, as well as the community's varied tendencies in licensing their works. To do so, Moilanen et al. created two data snapshots of Thingiverse from August and November 2013, and have released their datasets publicly to the world. Our research process led us to conduct our own webscraping processes, which has been built to match Moilanen et al.'s data structure. We provide an additional snapshot, from 2015, which can contribute to ongoing research built on the webscraping of popular 3D printing sites. This allows us to map out the growth of connections between objects in Thingiverse, and discover new categories of use. Furthermore, we have conducted a second webscraping process which approaches Thingiverse laterally, investigating not the connection of objects, but the connection of connections: we have generated a map of tags that allows us to examine how different methods of categorisation relate to each other on Thingiverse, and by doing so enact the ties of the community.

The research conducted here builds from the context of work previously undertaken by members of the research team that situates practices of 3D Printing. AUTHORS (2015) identifies $3 \mathrm{D}$ printing as a set of cultural practices that sit above, and also interpret, additive manufacturing technologies. As such there is not just one future but many for 3D printing as creative prototyping with experts demonstrates (AUTHORS 2013). Further, AUTHORS has argued that 3D printing could be a central component in a socio-technical transition or reconfigurations (AUTHORS 2012) and has used SNA to map patent citation data to 
visualize how key actors are nodes in cross-organisational relationships underpinning 3D printing's emergent ubiquity (AUTHORS 2013).

Identifying 3D printing as a practice, rather than a technology, allows our focus to foreground objects of creation, rather than people who create them. In this paper we adopt an optic situating 3D printing as a 'Technology' (with a capital t) centred on relationality, which affords a critical perspective on both technological 'hype' and 'determinism' (Mackenzie 2005). Following this Social Construction of Technology (SCOT) framed analysis, 3D printing cultures on Thingiverse are centred around the 'thing' or designed object on the site. 'Things' are the dominant point of action and engagement for users, to such a degree that users themselves recede into the background of the site. By mapping out the object-centred network of 3D printing cultures with social network analysis tools, we can see an aggregate model of user engagement with the current additive manufacturing technologies. As a more general project applicable to research outside of 3D printing, our work moves towards acknowledging how specific ways of organizing and representing metadata can have profound impacts on imagining the communities they enact. In this sense, we speculate on the extent that Object Oriented Ontologies can enable new ways of searching, examining, and analysing digital data within the humanities when people, concepts and things are interacting with their own agencies. Critiquing the user-centric ideal of Folksonomy, we tentatively introduce 'ontosonomy' as a way to signpost how people, objects and networks create and distribute digital order. We concretise the term here to energise its critique in the space of digital methodologies that pertain to humanities and social sciences. We hope readers can excuse our use of the neologism as we think it not only progresses, but nicely encapsulates the methodological issues that we came up against regarding the material reality interpreting of 'user-created' content online. 
Ontosonomy relates to the material reality of our methods for future researchers conducting studies on not only 3D printing, but any digital objects and the metadata that surrounds them. Our work engages with what Brügger and Finnemann (2013) call digital born materials. However, unlike Brügger and Finnemann's study that focussed on 'web materials' accessible through the level of the interface, we explore evolving collections of connected metadata to allow both synchronic and diachronic (spanning 2013 to 2015) insights to the relations between objects created by the Thingiverse community. To be clear, our methodology is not focussed on the 'users' that help make this community, although such methodological focus carved out for digital humanities is important (Warwick 2012). Instead, we generally follow Rogers (2013), to posit that the researcher must think through any corpus of data in its own digital terms, and adjust research strategies accordingly. A critical approach to methodological rigor and careful research design requires it. A critical approach also requires recognising that in the same way that the our objects of study are imbedded and constructed through their digital-relations, our own inquiry is built through and off these relations and then what the researcher is both able to and chooses to make visible. In other words, that the computational turn (Berry 2011) alters both methods of understanding reality, and methods of controlling that reality (techne) cannot be overlooked. Methodologically, our plan of action chooses to foreground metadata as a window of insight into the objects' use and the social meaning. This computational techne we employ enables us to perform analyses in aggregate, and assumes meaning can be found in how the data structures itself through the enabling constraints offered by the platform, input of the user, and the actant-like 'tag' links that make new connections visible for human members of the (research) community. Yet this is not to say that we set out to show how big data can replace or arise free from the regulating force of theory. On the contrary, we suggest that new theories, just as situated as the previous ones, may emerge based from the ways of knowing allowed by our methods. The extent that these 
new ways of knowing collapse other ways of knowing, is largely outside the scope of this article, but is a concern of any researcher who is able to identify his or her own assumptions (See Kitchin 2014 in relation to Big Data in the Humanities). Regardless, our perspective requires an epistemological stance that suggests individuals do not make meaning from objects in the world, but are placed within a 'world of meaning' (Crotty 1998: 79) made from a mélange of cultural networks and objects that shape thinking and behaviour. Including 'objects' signals a need to move past Crotty's original epistemological understanding of Constructionism via gesturing to an ontology that orients towards the object. We ask what happens when human conversations about meaning are secondary to the metadata connections between objects that make them visible and useful for human users in the first place.

This article predominantly addresses the methods by which we extract and analyse our data with an eye towards the concepts of tagging and remixing as they occur on Thingiverse, and critiques the linked methodological concerns that arise. This article will progress by historically contextualising what we claim are the key enablers for social-consumer practices of 3D printing, before moving to discuss our own webscraping process including our 'Tagscrape' and 'Thing-scrape', and finishing with theoretical reflection that positions our research to questions of digital research methodology. The purpose of this research then is to map tendencies of use of 3D printing for its own sake and as a way to elicit the need for new forms of media analysis focused on the ways metadata constitute and guide communities' communications and govern their potentials. Future research will reflect on how our current methodological choices interact with ongoing survey and interview data, that we hope can extend, contrast and provide triangulation of our findings.

\section{A Brief History of Socialising 3D Printing}


For this paper we identify three distinct phases in the gradual emergence of $3 \mathrm{D}$ printing into social life. The chronology of events here is by no means canonical, instead serving as a guide. Many of these phases arose in parallel or concert.

First, there is the pre-production industry phase the origin of 3D printing spans back to its uptake in pre-production prototyping - that is, so-called 'rapid prototyping': the creation of one-off models near to or by the creator (Kellock 1989). The inventor of 'stereolithography' (SLS), a process common to rapid prototyping, Charles 'Chuck' Hull went on to found 3D Systems (1988). The affordances of being able to fabricate models rapidly and costeffectively met the needs of form, fit and function trials (Wood 1990) as well as industrial designers, architects and engineers at the drawing board stage of bringing a product to market (Pham and Gault 1998).

Second, is the post-production industry phase. Advances in the additive manufacturing of metals and other materials made objects able to withstand stress and strain or with unique properties, geometries or aesthetics that enabled uses in part in, or whole for final, end-user products (Bak 2003). The inventor of one of the technical processes involved here, Fused Deposition Modelling (FDM) went on to found Stratasys. With these affordances in postproduction, 3D printing emerged as a viable tool for limited product runs for aerospace, automobile and military applications where customization and complex geometries trumped economies of scale (Hopkinson, Hague, and Dickens 2006).

Third, there is the peer-production phase. This affected both 'hardware' of printers and the 'software' of object design. The expiry of FDM patents enabled mass-market and open source 3D printers targeting hobbyists, enthusiasts and 'makers' beyond 'rapid prototyping' applications who enact peer-production (Moilanen and Vadén 2013). Entry into this market by rapid prototyping firms (eg. Stratasys, 3D Systems) initially produced little success. 
The firm Makerbot, utilizing the open source 'Reprap' blueprint, innovated by British academic Adrian Bowyer, struck a nerve in the media via claims of its printers being designed to be self-replicable and spawned much public interest in 3D printing. Later, social funding sites like Kickstarter offered space for multiple developments and mutations of 3D printer experiments. These ranged from firms that promised Apple-like vertical integration and ease of use and design within walled gardens of printable objects (see Pirate3D out of Singapore) and sub-\$100 printers that required user to bring their own tea cup for use as a print bed (see Peachy Printer out of Saskatchewan, Canada).

In 2008 Makerbot launched a 3D printing file repository named Thingiverse that allowed users to upload and remix designs. Thingiverse has grown into the largest public repository for 3D printable objects, with over a million objects shared as of mid-2015. However, the politics of what should be shared disaggregated practices of sharing. For instance, when Thingiverse removed firearms-related design files in late 2012, an individual named Cody Wilson moved to create defcad.com to serve as an unregulated repository of printable objects. Even the website The Pirate Bay opened a 'physibles' section to host torrents of 3D printable objects. These patterns were spurred onwards by increasingly easy to use CAD-like software that lowered barriers to $3 \mathrm{D}$ design, such that $3 \mathrm{D}$ printing was starting to be practiced less as a manufacturing process than as a form of play (de Smale 2014). As of 2015 over a dozen wellstocked 3D printing file repositories are found online, with varying implementations of intellectual property policies. Coming full circle, Thingiverse's parent Makerbot was bought by Stratasys in 2013 . Thingiverse now implements complex intellectual property agreements vertically between Thingiverse users and the company, that are separate and distinct from the sharing norms and licencing available horizontally between Thingiverse users (Moilanen et al. 2015). It is from the Thingiverse universe of shared objects that this paper now turns its analytical capacity. 


\section{Webscraping: Things and Tags}

Our research builds on the webscraping work by Moilanen et al. which investigated items on Thingiverse. A data-extraction barrier that Moilanen et al. encountered was that private objects are inaccessible to webscraping. Our research methods managed to get past this barrier in a limited fashion, and we report on the conclusions that we have drawn from this. However, our limited progression of technique does not speak past Moilanen et al.'s extensive analysis of licencing issues on Thingiverse, which uncovers important cultural practices around 3D printing. Instead, we open the way for new forms of analysis within the Thingiverse dataset.

A 'thing' is the name given to items kept on the Thingiverse website. For analytical purposes, it is a file or set of files associated with a unique serial number, and is intended for use in 3D printing. Each thing has a range of associated metadata including fields about authorship, origin, creation date, images, comments, familiar social data (likes, views, downloads), as well as self-reporting data about the number of times other uses have printed an object. It also includes two sets of optional data: a set of tags, and a reporting field about any items that the thing was 'remixed' from. Some tags are attached by the system in certain situations and others are user-created (and are thus subject to interpretation). User-created tags appear to be subject to an unknown degree of maintenance by Makerbot (abusive language does not generally appear amongst the tags).

Two different webscrapes were conducted as a component of the research. While Thingiverse does provide an API system for developers, the public-facing API is limited to smartphone and web-app development, primarily for updating and changing item names and tags, and providing comments on specific items. In this sense, the API is guided more towards individual engagement, and less towards research-appropriate methods. Because of this, 
bespoke webscraping tools were necessary to extract data. Like most online database access systems, Thingiverse uses a consistent page structure, which meant that webscraping tools are sufficient to extract desired data. Inevitably, both scrapes obtained an incomplete sample of the database, but are sufficiently coherent to the database at the time of their scraping for research to continue.

The first, 'Tag-Scrape,' aggregated tag counts from the Thingiverse website, and ran on 24 June 2015, gathering 42180 entries. This scrape obtained data regarding the names of tags and their frequency of use by the community. The method involved scraping the website using the 'kimono' plugin by Kimono Labs for Chrome. This plugin quickly creates exportable datasets with minimal programming and was used to address the top-level lists of tags on the site Thingiverse.com/tags, rather than aggregating from the individual item pages. After being extracted by kimono, the data was analysed and cleaned using a combination of computations administered through the Python and $\mathrm{R}$ programming languages. Because this method collects data on tags directly from the top level tag lists, including item counts, it still reported data on private-class objects. As such, this method obtained more information about the use of tags than 'Thing-scrape,' as mentioned below, due to the fact that, as Moilanen et al .note, approximately $42 \%$ of the database is private-class, and thus inaccessible to direct webscraping.

The predominant issue with our Tag-Scrape was that the Thingiverse tag browsing system has an error which limits its ability to display certain, seemingly arbitrary page numbers above certain values, which can be attributed to a lack of correspondence between the web interface and the database itself. The web interface appears to default to a range of 30 pages, and is not adept at displaying information after page 228. The maintenance of the tag search page appears to have been depreciated relative to other portions of the site for an unknown amount of time. Nevertheless, we obtained data on 42180 tags, while Thingiverse reported 
the existence of 42182 tags at the time of the scrape. Assuming the reporting data is correct, this indicates our scrape obtained $99.995 \%$ of tags.

The second, 'Thing-scrape,' obtained individual metadata from all publicly-accessible item pages on the Thingiverse website, and ran for two weeks from 16 to 30 July 2015, gathering 355867 entries from an unknown number of webpages. This scrape was conducted using Python scripts built on the BeautifulSoup package for dealing with HTTP requests, and the scripts were run on the Australian NeCTAR cloud server system for researchers. Thingiverse has a standardised item reference code, referred to as the 'ThingID.' This begins with the first publicly visible item, ThingID 2, and ends with the most recent object in the database possessing the highest item code. Item codes that do not refer to accessible objects have either being assigned a 'private' status, been subsequently deleted, or represent corrupt or damaged data.

Due to Thing-scrape addressing a far larger dataset, the scraping process was conducted on the Nectar cloud server system and was more complex than Tag-Scrape. Where the information on Tag-scrape was obtained from 1407 known webpages, the number of webpages with viable information was not known in advance.

The initial run of Thing-scrape possessed significant data loss. This data loss was attributed to internet packets that have been lost during multiple connections. As a result, 'Thingscrape' script was iterated multiple times in order to collect a saturated dataset, removing duplicated ThingID lines for each pass. A final test scrape was parsed, and only retrieved duplicate data. In all likelihood this suggests that the vast majority of the remaining data on the servers either had access restrictions or was in some way corrupted.

Our methodology removed one constraint to do with the visibility of private objects on Thingiverse, but only by the proxy of their associated metadata. This brings up two 
considerations for the practices of researchers when employing digital methodologies. First is that the limits set by APIs, interfaces, and protocols are more malleable then their digital nature suggests. Research skills, in part, are shifting to work within - and against - the limits present. While such ways of knowing allude to Mahrt and Sharkow's $(2013 ; 30)$ concern of a temptation to let applications or platforms determine the research question, our view is that in practice, such considerations are not so starkly binary. There is what we'll call here internal and external malleability for digital data. Internal factors of malleability can be thought of in a similar way to what Berry $(2011 ; 1)$ calls the 'plasticity' of digital objects. This is a key affordance of digital remediation that allows, in Berry's words, a 'folding' of reality to approach cultural objects and subjects in radically new ways. From our perspective, it allows a 'folding' of the digital objects themselves - here through the 'lateral' inquiry to using metadata attached to objects to define the relations between (hidden) objects, that then enabled speculation on the significance of how these objects relate to each other in cultural use. External malleability might better be explained through practices of hacking and combining ad-hoc discrete processes and digital pathways of data gathering methods, such as APIs, public data, and proprietary feeds to elicit ways of knowing that were previously unavailable. In our research plan, this meant running parallel processes to find and return data associated with specific objects in different ways, and using discrete platforms, protocols, and targets for extraction to eventually aggregate data for analysis in new ways. The ethics of hacking together disparate processes, or using computer-to-computer connections in ways that they were not imagined by their proprietors are complex and uncover evolving tensions in digital methodologies (see reports of the death of Aaron Swartz). Discussion on the ethics of hacking for research is beyond the scope of this article and we note that our own processes were cleared by the university ethics board. The second consideration involves what is made 
visible and the type of knowledge claims this engenders. Considerations of visibility epistemological basis are dealt with below in relation to analysis of our Tag-scrape.

\section{Tag-scrape}

Returning to our data, in agreement with Moilanen et al., the data suggests that the 'customized' tag was highly significant, appearing approximately 25 times more frequently than the next most common tag. The 'customized' tag is auto generated by internal mechanisms within Thingiverse when objects are modified using a built-in Thingiverse webapp 'customizer'. These modifications are generally very simple geometric multiplications. Let us take the 'Customizable Battery Tray' item, with ThingID \#48235. This item is a battery holder for AA batteries that can be modified to make the battery tray taller or shorter, or duplicated alongside itself to create a new object. This process is trivial, and simply involves moving a scroll bar on the screen. It requires no access to specialist software and in the researchers' experience, no other means of generating a new 3D printer file is as basic. As a result, the customized tag represents $37.7 \%$ of all tagging actions on Thingiverse. The customize tag shows the impotence of users on Thingiverse. Only through simple sliders do most objects allow themselves to be customized by the typical user. Further, any subsequent object made through this process is stained with `a scarlet letter of 'customized', denoting a hierarchy that the typical user cannot escape.

Our dataset diverges from Moilanen on two fronts: first, Thingiverse has had two years' worth of content added; second a different scraping method has drawn from different resources. In terms of variances, six out of the top ten tags have changed: 'photobooth', 'scan', 'scan0', 'scan1', 'scan2', and 'MakerBotDigitizer' identify the process of scanning real-world objects to produce printable file format that models the original object (see Table 
1). Investigating any of these tags shows that the vast majority of the items are non-public. For example, the tag 'Photobooth' was scraped with 16801 reported items. Investigating this tag on 7 October 2015 indicates that the tag has 16786 reported items, of which only 243 are visible. It is important to note that the tag count has reduced in the intervening period, perhaps indicating the de-tagging of items, or the deletion of entries.

[Table 1]

In the table above, the variations between the datasets are clear. Investigation of the different tags indicates that 'photobooth' is a tag that is automatically applied to items uploaded from Photobooth-branded hardware. We suspect photobooth references 3D Systems commercial “3Dme Photobooth" machines, as well as other systems targeted at users scanning themselves or other objects. Additionally, 'scan 0', 'scan 1', and 'scan 2' are tags automatically applied by Photobooth software to subsequent scans of a single object. Observation suggests that these are attempts to generate more accurate scans. We found no further tags using the 'scan \#' pattern, suggesting the high degree of similarity between these tags, in terms of the number of items hidden are a likely due to being a subset of the 'Photobooth' tag, and will likely have the same associated practices. The non-numbered 'scan' tag appears to be generically added by users for all forms of scans, and the 'MakerBotDigitizer' appears to be an automatically generated tag. It is notable that both the Photobooth and the MakerBot Digitizer hardware are owned by MakerBot, who also own the Thingiverse platform.

What should be highlighted is the fact that the high-privacy-rate tags are all used to describe the scanning in of real-world objects, specifically scans of people. 'Photobooth', 'scan', and 'MakerBotDigitizer' are tagged on items that are extremely likely to have restricted access. In 
fact, it is notable that a significant proportion of the remaining objects have an inverse relationship to privacy, when compared to scanned objects.

As Moilanen et al note, this data is inaccessible via direct webscrapes, as many of the items from these categories are set to private. Many of the remaining public items with the 'photobooth' tag are scanned images of people. While it is possible that there is a data reporting error on the site, we can hypothesise that many of these private files represent images of people, or that they represent otherwise personal objects, which individuals have since set to a private status to preserve access to their personal appearance or other items that require online privacy.

There are also many tags that reference only one object. Indeed, $42.0 \%$ of all tagged items only have one tag, yet this only represents $1.6 \%$ of the tags on the site. Thus $58 \%$ of the items hold $98.4 \%$ of the tags. This may indicate that not only is the long-tail well in effect, but many tags are not informative insofar as they do not generate a connection between two objects, and simply remain, for now, unique to a singular item. On the other hand, within Tag-scrape, we identified common error patterns common to user-entered text-based information. Many entries were ad-hoc, personalised, misspelled, or sequential. For instance, some object designers have used the tagging system to add their name to their creations, in addition to the author metadata field. In other cases, scans of individuals have also been tagged with the name of the individual scanned. In a few isolated cases, items are in languages other than English, perhaps underreporting overlap.

\section{Tag analysis}


Initial observations of tags revealed general categories of tags as per Table 2 . While analysis of these categories is ongoing, we are able to address some early observations: qualitative tags cut laterally across all manner of genres of item, as do the hardware and software tags; however, it can be speculated that qualitative tags are more likely to be added by the Thingiverse's community of users rather than the maker of the object. Dimensional information is listed because 3D printer files are generally relational point clouds, and can be modified to easily create different scales for printed objects. Further, tags allow users to identify objects designed to fit together at certain scales. Many of the branded category tags are designed as free replacements for those objects. Notable are the wargaming replacements, such as for the popular Warhammer brand of toys, as are the replacement LEGO pieces. As Angela Daly (2016) notes, these branded objects are consciously dealt with by amateur producers as having complex legal issues. Many of the other branded objects include things such as fake Starbucks mugs, printable Nike ‘swoosh' objects, or replacements for expensive camera accessories such as grips or housing.

[Table 2]

Tags, on Thingiverse, are a complex system that describes the relationship between objects, as managed both by authors, and the community at large. At times they speak of their origins, at other times they speak of their purpose. As seen in Figure 1, the breadth and centrality of tags other than 'customized' spans a horizon of use patterns that fade into the long tail of internet cultures. Further, tags are used for a variety of personal attachment: some are private, some public.

The limits of ways we can claim this knowledge are largely dependent on the metadata linking these objects - namely the emerging 'ontosonomy' of tags applied by things (like the 'photobooth' tag), people, and we'd argue, the networks themselves through the 'customize' 
tag and the penultimate form of visualisation of connections that enables researchers to claim the data is interpretable. On the one hand, ontosonomy references a certain type of 'object oriented' ontological triangulation. Technical objects, human users and data processes are organising data into knowable relations and specific patterns of use. On the other hand, these relations are knowable and accessible in different ways to different parties: through the Thingiverse web interface for users, but for researchers through our own chosen methodologies that illuminate new patterns and forces. In this way one might argue that the ways metadata constitute and guide communities' communications and govern their potentials is never fully visible. Instead, it presents a managed visibility (Flyverbom 2015), that is multivalent and imbedded within the political materiality of digital disclosure (Authors 2016). To emphasise this point and better understand the connection between objects that tags link to, we will move on to the 'Thing-scrape' analysis.

[Figure 1]

\section{Thing-scrape}

Thing-scrape is a dataset, for which analysis has only just begun. Two modes of social network analysis were conducted on this dataset, which were in part informed by the analysis of Tag-scrape. While SNA is often directed to identifying patterns of behaviour and use within large-scale social networks, in our case, we have used SNA to map out relationships between tags on Thingiverse. This allows insights to how tags tend to be used side-by-side, and also to map our hierarchies of remix. In terms of remix, we considered the frequency of remix per object, as per Moilanen et al, but also tried to understand what linkages form 
between different object groups. For tagging practices, we can start to map out connections between and across the different categories of use that we identified in Tag-scrape.

For the analysis of both remix items and tagging items in Thing-scrape we used the following for our method of SNA. First we identified metadata categories for analysis in Excel, and cleaned the data in order to remove null fields. Data was then exported to a CSV file for processing by Gephi, a network visualisation program. The open-ord algorithm was used, due to the speed which it processes large datasets, and the clean separations that it generates between different clusters in a network, allowing for clearer and smoother analysis of data.

In the case of our analysis of tags, an additional step had to be included before importing data to Gephi. Tags are scraped from Thingiverse as a comma-separated string containing an arbitrary number of variables, including zero. Thus any string had to be separated into a set containing multiple variables for analysis, and, furthermore, any set of tags which a size greater than 2 needed to be reconfigured into pairs. In other words, if a set was reported as [a, $b, c]$, our data was processed such that the set took the form of [a,b], [a, c], [b, c]. This change was processed in Python, and meant that the data could be processed by Gephi. Self-loops (nodes that connect to themselves) were removed to ensure that networks took priority in the graph. Additionally, because any set of size 0 or 1 holds no information about connections between variables, and thus no information about networks, these sets were discarded.

\section{Thing-analysis}

Remix is an internally reported quality within Thingiverse indicating that the current object is a descendent of another. Each item on the site may refer back to any number of other previously-added items on the site. This concept is obviously inspired by the cultural practice of remix, while falling short of being able to track all manner of types of remix, as explored below. 
Analysis of patterns of remix patterns identified several features. First, by far the most common form of remix was the modification of mobile phone cases. For many common models of phones (predominantly iPhone \& Samsung models), generic mobile case structures would be uploaded, which would then be remixed by users to add a personal accoutrement, name, or image to the case. However, this form of remix generally only led to one 'step' of evolution in the remix process. Users would generate their own custom phone case, and then there would be no subsequent evolution of that object-form. This is likely due to the personalised nature of these remixes - the strict utility for other users is less than the utility provided by the original generic phone case. As such, the initial SNA methods that attempted to use algorithms designed for cladistics stalled by the fact that there was very little complexity in the evolution path of objects. An additional reason for this low level of evolution is likely to be the fact that the Thingiverse customizer system allows for easy remix of a parent object, but the subsequent descendent objects do not possess the same capacity.

Second, lithopanes are also highly remixed. Lithopanes are opaque plastic sheets, which have images printed into them topographically. When put against a window or light source, the varying thickness of the print becomes semi-transparent, allowing an image to shine through. The item ThingID \#74322, which is one of the few non-phone case objects to have a high degree of remix, is a lithopane that allows users to import their own image. Between this, and the phone cases, it is clear that the most successfully remixed items are the most generic simple plastic plates, or simple phone cases, which can easily be modified to include some sort of personalised design.

Third, fonts are important, but not highly remixed directly. Fonts are one class of objects that had high centrality, but low levels of remix, meaning that while a font might not be remixed all that much itself, several font files have been used by objects which in turn become the 
basis for a large swathe of remixed items. In particular, the Braille font item ThingID\# 16193 has a degree of centrality that far exceeds any other item on the site.

Fourth, 'collections' generate artificial remix status, and generally do not lead to subsequent developments. A collection consists of a number of remixed objects packaged into one file, allowing for a single print job to produce a large number of objects. It also simplifies finding, for instance, the entirety of the available Pokémon figurines on the site. While collections represent less than $1 \%$ of the total number of remixed objects on the site, they do have a high degree of centrality within the network. Most objects (99\%) only declare one original object as their parent, collections, on the other hand, refer to many objects. As such, they represent a significant role within SNA, while not indicating much in terms of the idea of remix culture.

Finally, remix is an auto-generated state on the site, while remix as a cultural practice has no real reliance on didactic processes of reference, instead relying on cultural knowledge, inspiration, hybridity, and evolution. A key element to remix is the subtle elements that inhere in a text which suggest, rather than inform, about their origins (cite?). Thingiverse provides no datafield for the evolution or cultural influences that may have inspired users to create their objects. For instance, brands are tagged, not indicated as remixed. This means that Thingiverse does not report on remediation as a practice, but instead only as references between database items. Remix within Thingiverse is commonplace, yet often banal. The deprivileging of remix culture through interface and algorithm shows how visibility is controlled in ways more complex than a folksonomy, and suggests that the intellectual property concerns Moilen et. al discovered as baked-into Thingiverse are maintained by actants other than human intervention.

\section{Concluding theoretical reflections}


To conclude our discussion on the importance of objects, we turn to Bruno Latour's (1993: 142-145) frequent mention of the Parliament of Things, where people, concepts and things interact with their own agencies to create meaning, politics and knowledge. It seems apt, that the largest public file repository of 3D printing items where people, concepts and things interact to, ostensibly, materialise new things, is known as the 'Thingiverse'. We have shown that within this space the working of knowledge relations moves past the user-centric ideal of tag based folksonomies and might be better described as an ontosonomy. To speculate on how this works, we posit that digital things in Thingiverse as able to act in two ways: as the object itself and its metadata.

What can we say of the objects on Thingiverse? The performance of any such object is built when the digital file interfaces with another 'thing', a 3-D printer, allowing a complex performance in the printer's interplay of digital and material technologies. The human user plays a peripheral role here, for the most part witnessing but not participating, reduced to menial tasks such as loading consumables, and clicking 'print'. The end product here is yet another object - perhaps a iPhone case, which having been born of machine-agency can now take its place in intimate proximity to its reason d'etre, the iPhone; the new hybrid offering its own new formations of use.

Each object speaks somewhat of its origins: its author, its software, its inspiration, its future. What is apparent, however, is that it is the objects that speak of themselves. While users can and do comment on files and forums, and it is at the users' behest that these items emerge, the Thingiverse site is a site of objects speaking for themselves and for other objects. The hierarchy of the site privileges the object, or thing, as the space of communication. Forums exist, where users can discuss 3D printing, or different categories of use, but these are paratexts rather than integral; the site begins and ends with the individual objects. Things dominate the front page, they clamour for attention, and it is the objects and their interrelation 
to other objects (3D printers, Photobooth, Warhammer, scaling variables etc.) that objects are defined by, and that the users are ultimately interested in.

Yet tags are not only a mere second-tier means for users to try and understand what these objects are here to do. Tags do exist for humans in a world in which humans are not privileged, implying what these objects will do when they are communicated into a 3D printer and decanted into a real-world space. However, they also 'fold' their digital objects into networks of exploration and proliferation. They enact the long tail of user inconsequentiality, with single-tagged objects lost and unknowable in a database dependent on relations to objects. Tags thus assemble clumps and momentum of culture and meaning in ways that users are not always aware. Latour, in calling for a recognition of the already existing Parliament of Things requests that we ratify what has already been assembled. This is a part of what this project has started to map out: a relationship between the naïve, the critical, the parochial, the reflexive, and the automatic practices of tagging objects conducted by users and machines, and the potential for research methodologies to reflect upon object oriented ontologies. By granting value to the collected work of users and the objects they create, patterns of use and understanding emerge from the tagging of objects. 3D printing has a complex relationship towards human sense-making and culture, but importantly, it is the materiality that scholars are only just starting to ontologise. 


\section{Acknowledgements}

In order to preserve anonymity, acknowledgements and ethics approval codes will be fully detailed contingent on passing peer review.

\section{Bibliography}

\section{AUTHORS (5)}

Bak, David. 2003. "Rapid prototyping or rapid production? 3D printing processes move industry towards the latter." Assembly Automation 23 (4):340-345. doi: doi:10.1108/01445150310501190.

Berry, D.M., 2011. The computational turn: Thinking about the digital humanities. Culture Machine, 12(1).

Brügger, Niels, and Niels Ole Finnemann. 2013. "The Web and digital humanities: Theoretical and methodological concerns." Journal of Broadcasting \& Electronic Media 57.1: 66-80.

Crotty, M. 1998. The foundations of social research: meaning and perspective in the research process. London; Thousand Oaks.

de Smale, S. 2014. Building Material: Exploring Playfulness of 3D Printers. Transactions of the Digital Games Research Association, 1(3).

Daly, A. 2016 Printing Freedom: Socio-Legal Aspects of the 3D Printing Revolution, Palgrave Macmillan: (forthcoming)

Hopkinson, Neil, Richard J. M. Hague, and Phill M. Dickens. 2006. "Introduction to Rapid Manufacturing." In Rapid Manufacturing: An Industrial Revolution for the Digital Age, edited by Neil Hopkinson and Richard J. M. Hague, 1-4. Chichester: John Wiley $\&$ Sons.

Hull, Charles. 1988. "StereoLithography: Plastic prototypes from CAD data without tooling." Modern Casting 78 (8).

Kellock, Brian. 1989. "Excitement of technology trends." Machinery and Production Engineering 147 (3767).

Kitchin, R., 2014. Big Data, new epistemologies and paradigm shifts. Big Data \& Society, 1(1).Latour, B. 1993. We have never been modern. Cambridge: Harvard University Press.

Mahrt, M., \& Scharkow, M. 2013. The value of big data in digital media research. Journal of Broadcasting \& Electronic Media, 57(1), 20-33.

Mackenzie, Adrian. 2005. "Problematising the Technological: The Object as Event?" Social Epistemology 19 (4):381-399. doi: 10.1080/02691720500145589.

Moilanen, Jarkko, Angela Daly, Ramon Lobato, and Darcy Allen. 2015. "Cultures of sharing in 3D printing: what can we learn from the licence choices of Thingiverse users?" Journal of Peer Production (6):1-9.

Moilanen, Jarkko, and Tere Vadén. 2013. "3D printing community and emerging practices of peer production." First Monday 18 (8-5). doi: 10.5210/fm.v18i8.4271.

Pham, Duc Truong, and Rosemary S Gault. 1998. "A comparison of rapid prototyping technologies." International Journal of Machine Tools and Manufacture 38 (1011):1257-1287. doi: 10.1016/S0890-6955(97)00137-5. 
Rogers, R. 2013. Digital methods: MIT Press.

Wood, Lamont. 1990. "Rapid prototyping. Uphill, but moving." Manufacturing Systems 8 (12):14-18.

Warwick C. 2012. Studying users in digital humanities. Digital Humanities in Practice.:1-21.

Tables/Figures

\begin{tabular}{|c|c|c|c|c|c|c|}
\hline $\begin{array}{l}\text { Moilanen } \\
\text { top ten } \\
\text { (captured } \\
\text { late 2013) }\end{array}$ & $\begin{array}{l}\text { Reported } \\
\text { frequency }\end{array}$ & $\begin{array}{l}\text { Tag-scrape top ten } \\
\text { (captured late } \\
\text { 2015,variations from } \\
\text { Moilanen in bold) }\end{array}$ & $\begin{array}{l}\text { Tag-scrape } \\
\text { frequency }\end{array}$ & $\begin{array}{l}\text { October } \\
\text { investigation } \\
\text { of frequencies }\end{array}$ & $\begin{array}{l}\text { October } \\
\text { investigation } \\
\text { of visible } \\
\text { items }\end{array}$ & $\begin{array}{l}\text { Private- } \\
\text { class } \\
\text { items }\end{array}$ \\
\hline customized & 19206 & customized & 393533 & 463861 & 187915 & $59.5 \%$ \\
\hline $3 \mathrm{D}$ & 5286 & Photobooth & 16801 & 16786 & 243 & $98.6 \%$ \\
\hline Makerbot & 3757 & scan & 13838 & 15050 & 4366 & $71.0 \%$ \\
\hline Openscad & 3579 & MakerBotDigitizer & 11208 & 12365 & 2071 & $83.3 \%$ \\
\hline Useful & 2565 & $3 \mathrm{D}$ & 8299 & 8501 & 8343 & $1.9 \%$ \\
\hline Reprap & 2089 & openscad & 6473 & 6808 & 6686 & $1.8 \%$ \\
\hline Toy & 1933 & Scan 0 & 5802 & 5798 & 72 & $98.8 \%$ \\
\hline Parametric & 1671 & Scan 1 & 5517 & 5511 & 74 & $98.7 \%$ \\
\hline Model & 1536 & makerbot & 5443 & 5545 & 5432 & $2.0 \%$ \\
\hline holder & 1321 & Scan 2 & 5397 & 5389 & 86 & $98.4 \%$ \\
\hline
\end{tabular}

Table 1

\begin{tabular}{|l|l|l|}
\hline Category & Example tags & Apparent common use \\
\hline Dimension/geometric & $\begin{array}{l}\text { 3D, 2D, cube, Z-Axis, } \\
\text { 40mm }\end{array}$ & $\begin{array}{l}\text { Item designed with specific } \\
\text { ratios }\end{array}$ \\
\hline Representational & $\begin{array}{l}\text { Art, animal, moon, halo, } \\
\text { knot, scan }\end{array}$ & $\begin{array}{l}\text { Item's function is likely } \\
\text { representational }\end{array}$ \\
\hline Hardware & Makerbot replicator, reprap & $\begin{array}{l}\text { Item designed for specific } \\
\text { printer }\end{array}$ \\
\hline Software & $\begin{array}{l}\text { Sketchup, blender, } \\
\text { TinkerCAD }\end{array}$ & $\begin{array}{l}\text { Item designed using specific } \\
\text { software }\end{array}$ \\
\hline Date/time & 2013, 2014, July, Christmas & $\begin{array}{l}\text { Item produced on that date, } \\
\text { holiday-specific }\end{array}$ \\
\hline Printer materials & ABS, PLA & $\begin{array}{l}\text { Item intended to be printed } \\
\text { in this material }\end{array}$ \\
\hline Purposive & $\begin{array}{l}\text { Holder, screwdriver, sensor, } \\
\text { tensioner, food, wearable }\end{array}$ & $\begin{array}{l}\text { Item is designed as a } \\
\text { functional object. }\end{array}$ \\
\hline
\end{tabular}




\begin{tabular}{|l|l|l|}
\hline Qualitative/Affective/emotional & Cool, awesome, love & $\begin{array}{l}\text { Item evokes subjective } \\
\text { evaluation }\end{array}$ \\
\hline Brands & $\begin{array}{l}\text { Nike, Warhammer, Canon, } \\
\text { GoPro, iPhone, Arduino, } \\
\text { LEGO, Pokémon, }\end{array}$ & $\begin{array}{l}\text { Item mirrors the aesthetics } \\
\text { of these brands, or adds to } \\
\text { or replaces proprietary parts }\end{array}$ \\
\hline Subcultural & cookie, robot, baixar & $\begin{array}{l}\text { Tag has context to a specific } \\
\text { subcultural group that has } \\
\text { origins outside 3D printing }\end{array}$ \\
\hline
\end{tabular}

Table 2

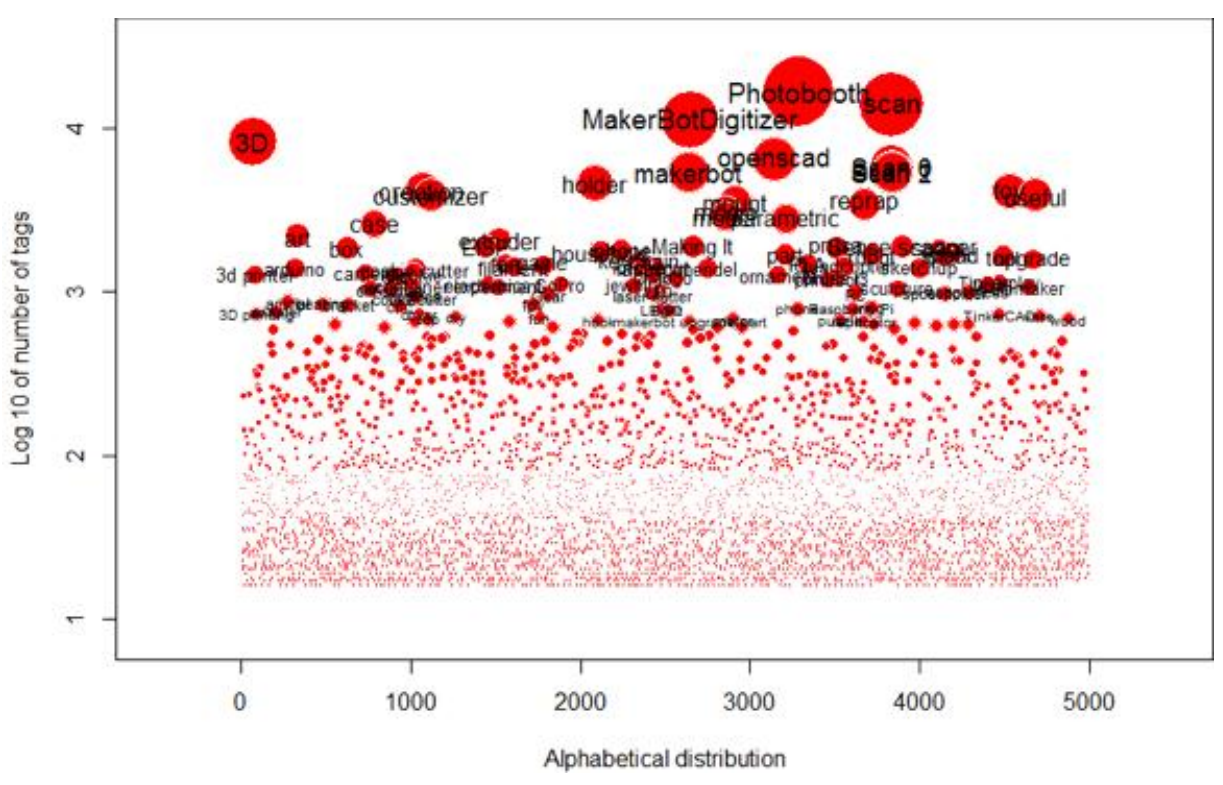

Figure 1 